\title{
Time-frequency analysis of the dispersion of Lamb modes
}

Journal of the Acoustical Society of America, Vol.105 (5), (May 1999), pp. 2669-2676.

\author{
W. H. Prosser and Michael D. Seale
}

NASA Langley Research Center, MS 231, Hampton, VA 23681-2199

Barry T. Smith

Norfolk Academy, 1585 Wesleyan Drive, Norfolk, VA 23502

Received

Accurate knowledge of the velocity dispersion of Lamb modes is important for ultrasonic nondestructive evaluation methods used in detecting and locating flaws in thin plates and in determining their elastic stiffness coefficients. Lamb mode dispersion is also important in the acoustic emission technique for accurately triangulating the location of emissions in thin plates. In this research, the ability to characterize Lamb mode dispersion through a time-frequency analysis (the pseudo Wigner-Ville distribution) was demonstrated. A major advantage of time-frequency methods is the ability to analyze acoustic signals containing multiple propagation modes, which overlap and superimpose in the time domain signal. By combining time-frequency analysis with a broadband acoustic excitation source, the dispersion of multiple Lamb modes over a wide frequency range can be determined from as little as a single measurement. In addition, the technique provides a direct measurement of the group velocity dispersion. The technique was first demonstrated in the analysis of a simulated waveform in an aluminum plate in which the Lamb mode dispersion was well known. Portions of the dispersion curves of the $\mathrm{A}_{0}, \mathrm{~A}_{1}, \mathrm{~S}_{0}$, and $\mathrm{S}_{2}$ Lamb modes were obtained from this one waveform. The technique was also applied for the analysis of experimental waveforms 
from a unidirectional graphite/epoxy composite plate. Measurements were made both along, and perpendicular to the fiber direction. In this case, the signals contained only the lowest order symmetric and antisymmetric modes. A least squares fit of the results from several source to detector distances was used. Theoretical dispersion curves were calculated and are shown to be in good agreement with experimental results.

PACS numbers: 43.35.Cg, 43.40.Le, 43.35.Zc, 43.20.Mv

Time-frequency analysis of Lamb modes

INTRODUCTION

In propagating through solid materials, acoustic waves may have velocities that are dependent on their frequency. This dispersion may be due to the material behavior, such as in viscoelastic materials. It can also be caused by the influence of specimen geometry on the wave propagation. This is the case for waves propagating in plates, rods, and shells. Accurate knowledge of this dispersion is important for many acoustic based nondestructive evaluation techniques. In ultrasonic testing, the velocity and its dispersion are used to determine the depth or location of flaws. It is also used in measurements of elastic properties. Related techniques such as laser generated ultrasonics and acousto-ultrasonics also require information about the dispersion for interpretation of data. In acoustic emission (AE), the velocity is needed to determine the location of the source of emission. As pointed out by Ziola and Gorman ${ }^{1}$, if not taken into account, highly dispersive propagation can lead to large errors in source location in AE testing.

In this study, the ability to determine group velocity dispersion of Lamb modes by using a time-frequency analysis method, the pseudo Wigner-Ville distribution (PWVD), was demonstrated. The technique was first applied to a simulated, broadband acoustic waveform in an aluminum plate. The technique was then applied to experimental waveforms from a unidirectional 
graphite/epoxy (AS4/3502) laminate. The broadband acoustic waves were generated by a pencil lead fracture (Hsu-Neilsen source) and were detected with broadband, contact, ultrasonic transducers. Measurements were made at several source-to-detector distances. A least squares fit was used to calculate the velocity for the lowest order symmetric and antisymmetric modes contained in the waveforms. Results are presented for propagation along, and perpendicular to, the fiber direction. Theoretical dispersion curves were also calculated and compared to the experimental measurements.

Time-frequency analyses such as the PWVD can offer several advantages for velocity dispersion measurements in comparison to more traditional techniques. For example, they can be applied to broadband signals so that as little as one measurement may be required to determine the velocity of multiple modes over a wide range of frequencies. This can be of significant importance when access time to specimens for measurements is limited, or when measurements are required for a large number of specimens. Another important advantage is the ability to analyze signals containing multiple propagation modes and/or reflections which superimpose and interfere in the time domain. These multiple modes and reflections can be separated in time-frequency space. The PWVD also provides a direct measurement of group velocity dispersion which may be the desired quantity in certain applications such as the recently developed AE source location technique from Ziola and Gorman ${ }^{1}$. Although, in theory, the group velocity can be obtained from phase velocity dispersion measurements, small measurement errors in the phase velocity can propagate into significant errors in the calculated group velocity.

For Lamb mode dispersion measurements, there is another advantage in that immersion of the plate is not required. Direct contact ultrasonic sensors can be used. This is in contrast to corner reflection and leaky Lamb measurement techniques which have been widely used by Mal et al. ${ }^{2}$, 
Chimenti and Nayfeh ${ }^{3}$, and Balasubramaniam and Rose ${ }^{4}$. These techniques also require a number of measurements with the wave incident to the plate at different angles to map out the dispersion curves.

The difficulty of phase unwrapping, which occurs in the Fourier phase dispersion measurement technique $e^{5-7}$, is not present in time-frequency based analysis. The Fourier phase technique also cannot be used when multiple modes or reflections are superimposed in time. However, another technique which overcomes these limitations is the two-dimensional Fourier transform method which was presented by Alleyne and Cawley ${ }^{8}$ and applied by Costley and Berthelot ${ }^{9}$. A difficulty of the two-dimensional Fourier transform technique is that a large number of closely spaced measurements is required to avoid aliasing when Fourier transforming from the spatial domain. However, instead of the Fourier transform, alternative spectral estimation techniques such as Prony's method can be used. A review of such techniques is provided by Kay and Marple ${ }^{10}$. Alternative spectral estimation techniques can be used when the spatial data is undersampled and thus, requires fewer measurements at different locations. However, these methods are somewhat susceptible to noise, requiring input data with high signal to noise level. Lang et al. ${ }^{11}$, Leslie and Randall ${ }^{12}$, and McClellan ${ }^{13}$ have applied these techniques to measure the dispersion of acoustic waves for geophysical applications.

More recently, another time-frequency analysis method, the short-time Fourier transform, has been used to characterize dispersion by Kwun and Bartels ${ }^{14}$. They evaluated the group velocity dispersion of the first and second modes of the axisymmetric longitudinal wave in cylindrical steel shells. The signals were generated and detected with magnetostrictive sensors and measurements made at frequencies below $100 \mathrm{kHz}$. 


\section{PWVD THEORY}

Time-frequency distributions provide a method for examining how the frequency content of a given signal changes as a function of time. The output of these distributions is the energy density, or intensity, of various frequency components of a signal at given points in time. Cohen ${ }^{15}$ has provided a detailed review of time-frequency distributions and discusses their numerous applications. Grade and Gram-Hansen ${ }^{16}$ compare the Wigner-Ville distribution to the short time Fourier transform (STFT) and wavelet transform (WT) for the analysis of nonstationary signals. It is pointed out that the Wigner-Ville distribution offers an advantage in that it is a more general transform which is not limited by the uncertainty relationship on simultaneous time and frequency resolution. However, this advantage comes at the expense of a couple of limitations. First, computational complexity is much greater for the Wigner-Ville distribution. Also, negative energy levels and cross terms may appear in the Wigner-Ville distribution which do not have physical meaning. This problem is discussed in more detail below.

From Cohen ${ }^{15}$, the general equation for a time-frequency distribution, $w(t, \omega)$, for an input time signal $\mathrm{s}(\mathrm{t})$ is given by

$$
\mathrm{w}(\mathrm{t}, \omega)=\frac{1}{2 \pi} \iiint \mathrm{e}^{-\mathrm{i} \theta \mathrm{t}-\mathrm{i} \tau \omega-\mathrm{i} \theta \mathrm{u}} \phi(\theta, \tau) \mathrm{s} *\left(\mathrm{u}-\frac{\tau}{2}\right) \mathrm{s}\left(\mathrm{u}+\frac{\tau}{2}\right) \mathrm{du} \mathrm{d} \tau \mathrm{d} \theta
$$

where the integrals are evaluated from $-\infty$ to $\infty$. In this equation, $s^{*}$ is the complex conjugate and $\phi(\theta, \tau)$ is an arbitrary function known as the kernel. Time and frequency are represented by $t$ and

$\omega$ respectively. For the Wigner distribution ${ }^{17}$, which is discussed in detail by Jeon and Shin ${ }^{18}$ and Wahl and Bolton ${ }^{19}$, the kernel function has a value of 1. Upon this substitution, Eq. (1) reduces to

$$
\mathrm{w}(\mathrm{t}, \omega)=\int \mathrm{s} *\left(\mathrm{t}-\frac{\tau}{2}\right) \mathrm{s}\left(\mathrm{t}+\frac{\tau}{2}\right) \mathrm{e}^{-\mathrm{i} \tau \omega} \mathrm{d} \tau
$$


For application to digitized or sampled signals, this equation must be modified to a discrete form. This is given by

$$
\mathrm{w}(\mathrm{m} \Delta \mathrm{t}, \mathrm{k} \Delta \omega)=2 \Delta \mathrm{t} \sum_{\mathrm{n}=0}^{2 \mathrm{~N}-1} \mathrm{~s}[(\mathrm{~m}+\mathrm{n}) \Delta \mathrm{t}] \mathrm{s} *[(\mathrm{~m}-\mathrm{n}) \Delta \mathrm{t}] \mathrm{e}^{\frac{-\mathrm{i} 2 \pi \mathrm{nk}}{2 \mathrm{~N}}}
$$

where $\Delta \mathrm{t}$ is the sampling interval and $\Delta \omega=\pi /(2 \mathrm{~N} \Delta \mathrm{t})$. The discrete Wigner distribution has several characteristics which limit its usefulness in this form. The first is a higher sampling requirement than the conventional discrete Fourier transform (DFT) to avoid aliasing. According to Boashash $^{20}$, the sampling frequency must be four times that of the highest frequency content of the signal as opposed to the usual Nyquist criteria for the DFT which requires it to be only twice that of the highest frequency content. Other considerations that are discussed by Jeon and Shin ${ }^{18}$ include the fact that the frequency resolution is only $1 / 4$ of that obtained by the DFT. Additionally, the Wigner distribution produces complicated and unexpected results when more than one frequency component is contained in a signal. "Noise," or the appearance of signal in the Wigner distribution at frequencies and times not actually contained in the waveform, is produced. It is caused by interference which consists of cross terms from the multiple frequency components and it makes interpretation of results very difficult. The final undesirable characteristic is that this distribution may take on negative values which do not have physical meaning. This most often occurs as a result of the interference discussed above.

A couple of solutions are available to overcome the first limitation, that is, the more restrictive sampling frequency requirement. The first and most obvious is to sample at the higher frequency. Hardware limitations in some situations may make this impractical. Also, once the data has been acquired at a given sample rate, it may not be possible to reacquire it at a faster rate. Thus, another solution was proposed by Ville ${ }^{21}$ and is discussed by Jeon and Shin $^{18}$ and Boashash ${ }^{20}$, among oth- 
ers. This approach involves the calculation of the Wigner distribution using the analytic signal. The analytic signal is a complex signal where the real component is the original signal and the imaginary component is its Hilbert transform. When the Wigner distribution is performed on the analytic signal, the requirement to avoid aliasing is now reduced to that of the Nyquist criteria. The resulting time-frequency distribution is known as the Wigner-Ville distribution. Boashash ${ }^{20}$ provides an intuitive rational on why this approach reduces the sampling requirement based on the fact that a single sample of the analytic signal provides two effective samples (the real and imaginary parts) of the original signal.

Another approach is used to minimize the effects of interference terms or "noise" in the distribution. This is the application of smoothing to the distribution. According to Jeon and Shin ${ }^{18}$, this emphasizes deterministic components and reduces those due to interference. A Gaussian window function, $\mathrm{G}(\mathrm{t}, \omega)$, is convolved with the Wigner-Ville distribution resulting in the Pseudo Wigner-Ville distribution, where

$$
G(t, \omega)=\frac{1}{2 \pi \sigma_{t} \sigma_{\omega}} e^{-\left(\frac{t^{2}}{2 \sigma_{t}^{2}}+\frac{\omega^{2}}{2 \sigma_{\omega}^{2}}\right)} .
$$

It has been shown that if $\sigma_{\mathrm{t}}^{2}$ and $\sigma_{\omega}^{2}$ are both greater than zero and if

$$
\sigma_{t} \sigma_{\omega} \geq \frac{1}{2}
$$

then the Pseudo Wigner-Ville distribution will contain only positive values. For application to the discrete Wigner-Ville distribution, the Gaussian window function is sampled over the region $\pm 2 \sigma_{t}$ and $\pm 2 \sigma_{\omega}$. It is given by

$$
\mathrm{G}(\mathrm{p}, \mathrm{q})=\frac{1}{2 \pi \mathrm{j} \mathrm{k} \Delta \mathrm{t} \Delta \omega} \mathrm{e}^{-\left(\frac{\mathrm{p}^{2}}{2 \mathrm{j}^{2}}+\frac{\mathrm{q}^{2}}{2 \mathrm{k}^{2}}\right)}
$$


where $\mathrm{p}$ and $\mathrm{q}$ are integer values that range over $\pm 2 \mathrm{j}$ and $\pm 2 \mathrm{k}$ respectively. The resulting sampled Pseudo Wigner-Ville distribution, $\mathrm{w}^{\prime}(\mathrm{r}, \mathrm{m})$, is given by

$$
\mathrm{w}^{\prime}(\mathrm{r}, \mathrm{m})=\frac{\Delta \mathrm{t} \Delta \mathrm{w}}{2 \pi} \sum_{\mathrm{p}=\mathrm{r}-\mathrm{j}}^{\mathrm{r}+\mathrm{j}} \sum_{\mathrm{q}=\mathrm{m}-\mathrm{k}}^{\mathrm{m}+\mathrm{k}} \mathrm{w}(\mathrm{p}, \mathrm{q}) \mathrm{G}(\mathrm{p}-\mathrm{r}, \mathrm{q}-\mathrm{m})
$$

\section{DISPERSION FROM A SIMULATED WAVEFORM}

The ability to measure Lamb mode dispersion from time-frequency analysis was first tested on a simulated acoustic waveform ${ }^{22}$. A finite element method, developed by Hamstad and Gary 23,24 for the prediction of acoustic emission signals in plates, was used to generate the simulated signal. A two-dimensional finite element formulation was used to model a surface loading source at the center of a circular plate. The radius of the plate was $0.5715 \mathrm{~m}$, the thickness was $6.35 \mathrm{~mm}$, and the receiver was at a distance of $0.254 \mathrm{~m}$ from the source. The force time response was linear with a rapid rise time of $0.1 \mu \mathrm{s}$, rising to a constant peak value of $1 \mathrm{~N}$. The material properties of aluminum from Kolsky ${ }^{25}$ were used. These were a density of $2700 \mathrm{~kg} / \mathrm{m}^{3}$, longitudinal wave velocity of $6320 \mathrm{~m} / \mathrm{s}$, and shear wave velocity of $3100 \mathrm{~m} / \mathrm{s}$. In the finite element model, 100 cells were used through the plate thickness providing an equi-axial cell size of $0.0635 \mathrm{~mm}$. The resulting simulated signal was sampled at $10 \mathrm{MHz}$.

As discussed by Gorman and Prosser ${ }^{26}$, sources normal to the plate surface, such as that modeled in this calculation, and the pencil lead break used in the experiments, produce signals with very large, low frequency $\mathrm{A}_{0}$ mode components. These large amplitude components make it difficult to detect and analyze the higher frequency, smaller amplitude, components of other modes. In the analysis of the simulated waveform, a $20 \mathrm{kHz}$ highpass Bessel (fourth order) digital filter was 
used to reduce these large amplitude, low frequency components. The early portion of this filtered waveform is shown in Fig. 1. The full waveform is shown in Fig. 2 along with the corresponding PWVD image which is discussed later. Arriving first in the simulated waveform are the lower frequency components of the $\mathrm{S}_{0}$ mode. The larger amplitude, lower frequency component of the signal is that of the $A_{0}$ mode. Superimposed on these modes are components of the $A_{1}$ and $S_{2}$ modes, as well as a reflection of the $S_{0}$ mode from the outer edge of the plate.

The original Fortran source code for the PWVD calculation from Jeon and $\operatorname{Shin}^{18}$ was modified for these dispersion measurements. The maximum number of points in the input signal and the maximum number of points in the calculated distribution were increased to provide enhanced time and frequency resolution. Zero padding of the signal was used to increase the frequency resolution of the PWVD calculation with 1024 zeroes padded onto the front of the signal. Additional zeroes were padded onto the end of signal to bring the total number of points to 4096. The PWVD, calculated from the 4096 point waveforms, provided 1024 X 1024 point resolution in the time-frequency domain. The smoothing parameters for the PWVD calculation, $\mathrm{j}$ and $\mathrm{k}$ from Eq. (6), were set based on the criterion that the distribution have no negative values. This occurs when

$$
\mathrm{j} \cdot \mathrm{k} \geq \frac{\mathrm{P}}{\pi}
$$

where $\mathrm{P}$ is the number of points in the original waveform. A square smoothing window was used with $\mathrm{j}$ and $\mathrm{k}$ both set equal to 37 to satisfy this equation.

The resulting three dimensional data of the distribution can be visualized in a number of ways. It can be displayed as contour plots, 3D surface plots, or as grey-scale or false color images. The latter approach was used in this work, where the color at a given $\mathrm{x}$ and $\mathrm{y}$ point in the image represents the amplitude of the distribution at a particular time and frequency. Due to the large 
amplitude difference between the different modes, the values were first compressed, by raising all values to the $1 / 4$ power, to more clearly show the multiple modes in the same image. An image processing program (NIH Image) was then used to adjust the contrast to provide the best visualization of the image data. The resulting PWVD image for the simulated acoustic signal in aluminum is shown in Fig. 2. along with the original time domain waveform. The different modes are labeled in the PWVD image.

For a given mode of propagation, the arrival time was determined as a function of frequency from the corresponding peak in the PWVD. However, there were several factors which affected the accuracy of this peak determination. First, for a given frequency, there were often several peaks in the PWVD, which corresponded to the arrivals of different modes of propagation. These peaks sometimes had significantly different amplitudes. A simple, amplitude based peak determination routine was insufficient for these calculations. Instead, an image processing approach was used to initially determine the approximate value of peaks at different frequency points for a given mode. A point and click selection tool (in NIH Image) was used to obtain approximate peak locations at selected frequency points. These frequency and time values were saved into a text file which was then used as input for a peak determination program. In the peak determination program, linear interpolation was used to provide an estimate of the peak times for all of the frequency values between those selected with the image processing program. In the PWVD image for the simulated waveform in Fig. 2, the peak values selected for the S0 mode along with the linear interpolations between the points are also shown. Then, for each mode and at each frequency point, a simple peak search routine was used to find the time for the actual peak within a predefined neighborhood of the approximate value. For these calculations, a window of \pm 15 points in time $( \pm 6 \mu \mathrm{s})$ was used to define the allowable neighborhood for the peak search routine. 
Another problem in determining the time for the peak was the limited time resolution of the PWVD. The reduction from the 4096 point, $0.1 \mu$ s sampled waveform, to 1024 points in time resulted in a resolution of only $0.4 \mu$ s for the PWVD. To improve the resolution in determining the peak, and thus the arrival time and resultant group velocity, a seven point cubic spline fit was used. The final problem in peak determination was background "noise" in the PWVD calculation. Smoothing reduced, but did not entirely eliminate, the problem of cross terms from multiple frequency components. This background "noise" made the peak determination more difficult, particularly for smaller amplitude signal components.

The group velocity dispersion was then calculated using the measured peak times (i.e. arrival times) as a function of frequency for the different modes, together with the known propagation distance. For the simulated waveform, the propagation distance was known exactly with no experimental uncertainty. The results are plotted in Fig. 3, along with the known group velocity dispersion curves for these modes in aluminum. As can be seen in this figure, the agreement between the PWVD calculated dispersion and theoretical curves for the $\mathrm{S}_{0}$ and $\mathrm{A}_{0}$ modes was excellent. For the $\mathrm{A}_{1}$ and $\mathrm{S}_{2}$ modes, the agreement was not quite as good. The effect of the background noise in the PWVD calculation, together with the much smaller amplitudes of these modes, resulted in larger discrepancies between calculated and theoretical velocities.

\section{EXPERIMENTAL RESULTS FOR A COMPOSITE PLATE}

The time-frequency dispersion measurement technique was further demonstrated with experimental measurements in a graphite/epoxy composite plate. Although this technique can be used on a single waveform as demonstrated in the previous section, these experimental results were obtained from several measurements. The added uncertainty in position measurements, along with 
the already coarse time determination in the PWVD analysis, provided the motivation for using additional measurements. Otherwise, the same image processing based analysis for approximate peak determination, followed by peak searching with a cubic spline fit was used.

The measurements were performed on a 16 ply unidirectional plate of AS4/3502 graphite/ epoxy. The nominal plate thickness was $2.26 \mathrm{~mm}$ with lateral dimensions of $0.508 \mathrm{~m}$ along the fiber direction $\left(0^{\circ}\right.$ direction $)$ and $0.381 \mathrm{~m}$ along the $90^{\circ}$ direction. Signals were generated by fracturing a $0.5 \mathrm{~mm}$ diameter pencil lead on the surface of the plate (Hsu-Neilsen source). This source mechanism produces broadband, transient acoustic waves and is often used to simulate acoustic emission signals. Fig. 4 shows a typical waveform produced by this source mechanism in the thin composite plate. The distance of propagation from source to receiver for this signal was $12.1 \mathrm{~cm}$ along a direction perpendicular to the fibers. Identified are the lowest order symmetric and antisymmetric Lamb mode components. No higher order modes were observed in the experimental waveform because of the smaller thickness plate used and the large attenuation of high frequency acoustic waves in this composite material.

Another factor which may have prevented observation of higher order modes was the large aperture (1.27 $\mathrm{cm}$ diameter) of the sensor used. The sensor was a 3.5 MHz ultrasonic sensor (Panametrics V182). This sensor is heavily damped to optimize its performance in thickness gaging in its intended use. It was operated far off resonance in detecting these signals which had maximum frequency contents below $500 \mathrm{kHz}$. In this mode of operation, this sensor provides high fidelity, flat frequency, displacement sensitive response. This has been demonstrated by $\operatorname{Prosser}^{27}$ by comparing its response to a wide band, displacement sensitive, optical interferometer. Gorman ${ }^{28}$ and Ziola and Gorman ${ }^{1}$ have previously used a similar type of $5 \mathrm{MHz}$ sensor for studying plate mode AE signals. Papadakis ${ }^{29}$ discussed why this type of sensor provides high fidelity, flat frequency 
response when operated off resonance. However, the phase cancellation across the large aperture of the sensor can limit its response at higher frequencies. Point contact sensors such as the pinducer and the NIST conical sensor do not suffer from this limitation, but were not available for these experiments. In addition the overall sensitivity of these point contact sensors is typically less than those with larger sensor area.

The signals were digitized at a sampling frequency of $10 \mathrm{MHz}$ with 12 bit vertical resolution (Digital Wave Corporation F4012). The 2048 point waveforms were padded with 1024 zeros in front of the signal to increase the frequency resolution of the PWVD. Starting at the last zero crossing of the experimental signals, the remaining values were set to zero to eliminate a big discontinuity at the end of the waveform. Such a discontinuity would provide an appearance of high frequency signal content in the PWVD images. Padding these zero values towards the end of the waveform also eliminated the reflection components of the $\mathrm{A}_{0}$ mode. However, the signals still contained very small amplitude $S_{0}$ reflection components. An additional 1024 zeroes were also padded onto the end of the waveform to increase the number of points to 4096.

To improve accuracy, seven measurements were taken at different distances of propagation in $1.27 \mathrm{~cm}$ increments over a range of $8.89 \mathrm{~cm}$ to $16.51 \mathrm{~cm}$. A least squares fit of arrival time versus distance was used to calculate the velocity. The source position was kept fixed for all measurements and the receiver was moved to different positions. At the largest distance, the source and receiver were centered in the plate. Waveform acquisition was triggered by a narrow band, 150 KHz resonant sensor (Physical Acoustics Corporation R15) positioned adjacent to the source.

Images of the PWVD along with the corresponding time domain signals for two distances of propagation along the $90^{\circ}$ direction are shown in Fig. 5. The large dispersion of the $\mathrm{A}_{0}$ mode is clearly seen in both the time domain signals and the PWVD images. The measured Lamb mode 
dispersion results for propagation perpendicular to the fiber direction are shown in Fig. 6. The uncertainties in the measured velocity values as determined from the uncertainty of the slope in the least squares fit are displayed as error bars on the measured data. Theoretical Lamb mode phase and group velocity dispersion curves for these anisotropic, laminated composite plates were generated using a method from Dong and Huang ${ }^{30}$ and Datta et al. ${ }^{31}$ For these theoretical dispersion calculations, nominal material properties, which were obtained from the manufacturer and are listed in Table I, were used. Comparison with theoretical curves shows good agreement, particularly for the $\mathrm{A}_{0}$ mode. The measured values for the $\mathrm{S}_{0}$ mode are slightly higher than predicted. Similar results were obtained in phase velocity measurements in this material in an earlier study by $\operatorname{Prosser}^{6,27}$. A possible explanation for this slight discrepancy is that the nominal material properties obtained from the manufacturer and used in the theoretical calculations are somewhat different from those of the actual material. Material property variations are not uncommon in graphite/ epoxy and may be caused by fiber volume variations, differences in cure processing conditions, and variations in resin chemistry.

The results for propagation along the fiber direction are shown in Fig. 7. Comparison with theoretical curves again shows good agreement for the $A_{0}$ mode. For the $S_{0}$ mode, the measured values in this case are slightly lower than predicted. These results are again consistent with the previous phase velocity measurements ${ }^{6,27}$ which showed good agreement between theory and experiment for the $\mathrm{A}_{0}$ mode, and slightly elevated measured values for the $\mathrm{S}_{0}$ mode. Again, the discrepancy was attributed to variations in actual material properties from the nominal properties used in the theoretical calculations. 


\section{CONCLUSIONS}

A time-frequency analysis method using the Pseudo Wigner-Ville Distribution (PWVD) was developed to characterize Lamb mode group velocity dispersion from broadband acoustic signals in plates. The technique was first demonstrated on a simulated waveform. This simulated signal was generated with a two-dimensional, dynamic finite element method to model propagation in an isotropic, aluminum plate. The simulated waveform contained multiple Lamb modes, as well as a reflection from a boundary, which were superimposed in the time domain waveform. The PWVD was able to separate these modes in time-frequency space to allow dispersion measurements. In this analysis, the arrival times, as a function of frequency, for the different modes are determined from the times of the corresponding peaks in the PWVD. These peak times were first estimated using an image processing program. Then the actual peak times were determined with a peak detection routine based on the image processor based estimates. The resolution of peak time determination was then further improved with a cubic spline fit to the peak. The arrival times were then used with the known propagation distance to calculate the group velocity dispersion. From the simulated waveform, portions of the group velocity dispersion for the $\mathrm{S}_{0}, \mathrm{~A}_{0}, \mathrm{~A}_{1}$, and $\mathrm{S}_{2}$ Lamb modes were determined. The agreement between the time-frequency measured dispersion and the known theoretical dispersion curves for aluminum was excellent for the $\mathrm{S}_{0}$ and $\mathrm{A}_{0}$ modes. The agreement for the $A_{1}$ and $S_{2}$ modes was adequate, but less than that for the other two modes. The amplitude of the $A_{1}$ and $S_{2}$ modes was much smaller in the time domain signal and the resulting PWVD. The peak detection was therefore much more susceptible to "noise" in the PWVD. This "noise" is the result of the interaction of multiple frequency components in the time-frequency calculation which is reduced, but not entirely eliminated, by the smoothing used in the PWVD calculation. 
The time-frequency dispersion measurement technique was further demonstrated on experimental data. Broadband acoustic signals were acquired in anisotropic, unidirectional graphite/ epoxy composite plates. Measurements were made for propagation both along, and perpendicular to the fiber direction. A pencil lead fracture (Hsu-Neilsen source) on the surface of the plate was used to generate the acoustic signals. Broadband contact ultrasonic sensors were used to acquire the waveforms. The signals contained only the lowest order symmetric and antisymmetric Lamb modes. The same process was used to determine the arrival times for these two modes from the peaks in the PWVD. However, in this case, a least squares fit from a number of propagation distances was used to calculate the dispersion. The added uncertainty in the distance measurement, along with the limited time resolution for the PWVD analysis, was the motivation for using the least square approach. Higher frequency sampling of the waveform could be used to increase the time resolution. However, the resulting increased number of points would significantly increase the time required for the PWVD calculation. The measured dispersion was compared with theoretical predictions. The agreement was good with discrepancies consistent with previous phase velocity dispersion measurements in this material. These discrepancies have been attributed to a lack of knowledge of the exact material properties for this composite. The nominal properties from the manufacturer were used in the theoretical dispersion calculations. It is widely known that actual material properties for a composite can vary as a result of a number of factors including resin chemistry variations, different processing conditions, and different fiber volume ratios.

Dispersion measurement from time-frequency analysis can offer a number of advantages in comparison to more traditional velocity measurement techniques. The first is that when a broadband acoustic source is used, few measurements, possibly only one, may be required to characterize dispersion for multiple modes over a wide frequency range. This can be particulary important 
when access time to specimens is limited, or when testing of a large number of specimens is required. The ability to analyze complex waveforms in which multiple modes and/or reflections are superimposed is also an advantage. Additionally, this method provides a direct measurement of the group velocity dispersion which is sometimes the desired quantity. Significant errors can occur when calculating group velocity from measured phase velocity data.

\section{ACKNOWLEDGMENTS}

The authors would like to acknowledge Jae-Jin Jeon and Young S. Shin of the Department of Mechanical Engineering, Naval Postgraduate School, who provided the original computer source code for performing the Pseudo Wigner-Ville Distribution. The authors would also like to acknowledge the efforts of William Winfree of the Nondestructive Evaluation Science Branch at NASA Langley Research Center who provided computer code which aided in determining the peaks of the distributions and displaying the images of the Pseudo Wigner-Ville distributions. Dr. Winfree also provided useful editorial comments in the preparation of this manuscript.

\section{REFERENCES}

1 S. M. Ziola and M. R. Gorman, "Source Location in Thin Plates Using Cross-Correlation," Journal of the Acoustical Society of America, 90 (5) 2551-2556 (1991).

2 A. K. Mal, C. -C. Yin, and Y. Bar-Cohen, "The Influence of Material Dissipation and Imperfect Bonding on Acoustic Wave Reflection from Layered Solids," Review of Progress in Quantitative Nondestructive Evaluation, 7B 927-934 (1988).

3 D. E. Chimenti and A. H. Nayfeh, "Leaky Lamb Waves in Fibrous Composite Laminates," Journal of Applied Physics, 58 4531-4538 (1985). 
K. Balasubramaniam and J. L. Rose, "Physically Based Dispersion Curve Feature Analysis in the NDE of Composites," Research in Nondestructive Evaluation, 3 41-67 (1991).

W. Sachse and Y. H. Pao, "On the Determination of Phase and Group Velocities of Dispersive Waves in Solids," Journal of Applied Physics, 49 (8) 4320-4327 (1978).

W. H. Prosser and M. R. Gorman, "Plate Mode Velocities in Graphite/Epoxy Plates,” Journal of the Acoustical Society of America, 96 (2) 902-907 (1994).

N. A. Schumacher, C. P. Burger, and P. H. Gien, "A Laser-Based Investigation of HigherOrder Modes in Transient Lamb Waves," Journal of the Acoustical Society of America, 93 (5) 2981-2984 (1993).

$8 \quad$ D. Alleyne and P. Cawley, “A Two-Dimensional Fourier Transform Method for the Measurement of Propagating Multimode Signals," Journal of the Acoustical Society of America, 89 (3) 1159-1168 (1991).

9 R. D. Costley, Jr. and Y. H. Berthelot, “A Laser Based Technique to Resolve Mode Propagation of Lamb Waves in Plates," Second International Conference on Acousto-Ultrasonics: Acousto-Ultrasonic Materials Characterization, 85-94 (June 24-25, 1993).

10 S. M. Kay and S. L. Marple, Jr., "Spectrum Analysis - A Modern Perspective,” Proceedings of the IEEE, $\mathbf{6 9}(11)$ 1380-1419 (1981).

11 S. W. Lang, A. L. Kurkjian, J. H. McClellan, C. F. Morris, and T. W. Parks, "Estimating Slowness Dispersion from Arrays of Sonic Logging Waveforms," Geophysics, 52 (4) 530544 (1987).

12 H. D. Leslie and C. J. Randall, "Multipole Sources in Boreholes Penetrating Anisotropic Formations: Numerical and Experimental Results," Journal of the Acoustical Society of America, 91 (1) 12-27 (1992). 
J. H. McClellan, “Two-Dimensional Spectrum Analysis in Sonic Logging,” IEEE ASSP Magazine 12-18 (July, 1988).

H. Kwun and K. A. Bartels, "Experimental Observation of Wave Dispersion in Cylindrical Shells via Time-Frequency," Journal of the Acoustical Society of America, 97 (6) 3905-3907 (1995).

15 L. Cohen, “Time-Frequency Distributions - A Review," Proceedings of the IEEE, 77 (7) 941-981 (1989).

16 S. Grade and K. Gram-Hansen, "The Analysis of Nonstationary Signals," Sound and Vibration, 30'th Anniversary Issue, 40-46 (1997).

17 E. Wigner, "On the Quantum Correction for Thermodynamic Equilibrium," Physics Review, 40 749-759 (1932).

18 J. Jeon and Y. S. Shin, "Pseudo Wigner-Ville Distribution, Computer Program and its Applications to Time-Frequency Domain Problems," Naval Postgraduate School Report NPSME-93-002 (1993).

19 T. J. Wahl and J. S. Bolton, "The Application of the Wigner Distribution to the Identification of Structure-Borne Noise Components,” Journal of Sound and Vibration, 163 (1) 101122 (1993).

B. Boashsah, "Note on the Use of the Wigner Distribution for Time-Frequency Signal Analysis," IEEE Transactions on Acoustics, Speech, and Signal Processing, 36(9) 1518-1521 (1988).

21 J. Ville, "Theorie et Applications de la Notion de Signal Analytique," Cables et Transmission, 2a(1), 61-74 (1948).

22 M.A. Hamstad, Private Communication, (1998). 
J. Gary and M. A. Hamstad, "On the Far-field Structure of Waves Generated by a Pencil Lead Break on a Thin Plate, J. Acoustic Emission, 12 (3-4), 157-170 (1994).

M. A. Hamstad, J. Gary, and A. O’Gallagher, "Far-field Acoustic Emission Waves by ThreeDimensional Finite Element Modeling of Pencil-Lead Breaks on a Thick Plate,’ J. Acoustic Emission, 14 (2), 103-114 (1996).

M. R. Gorman and W. H. Prosser, "AE Source Orientation by Plate Wave Analysis,” Journal of Acoustic Emission, Vol. 9(4), (1991) pp. 283-288.

W. H. Prosser, "The Propagation Characteristics of the Plate Modes of Acoustic Emission Waves in Thin Aluminum Plates and Thin Graphite/Epoxy Composite Plates and Tubes," NASA Technical Memorandum 104187, (November, 1991).

M. R. Gorman, "Plate Wave Acoustic Emission,” Journal of the Acoustical Society of America, 90 (1) 358-364 (1990).

29 E. P. Papadakis, "Broadband Flaw Detection Transducers: Application to Acoustic Emission Pulse Shape and Spectrum Recording Based on Pulse Echo Response Spectrum Corrected for Beam Spreading,” Acoustica, 46 293-298 (1980).

30 S. B. Dong and K. H. Huang, "Edge Vibrations in Laminated Composite Plates," Journal of Applied Mechanics, 52 433-438 (1985).

31 S. K. Datta, A. H. Shah, and W. Karunasena, "Wave Propagation in Composite Media and Material Characterization,” Elastic Waves and Ultrasonic Nondestructive Evaluation, S.K. Datta, J.D. Achenbach, and Y.S. Rajapakse, eds., 159-167 (Elsevier Science Publishers B.V., North-Holland 1990). 
TABLE I: Properties of AS4/3502 used in Theoretical Dispersion Calculations

\begin{tabular}{|c|c|}
\hline $\begin{array}{c}\text { Elastic } \\
\text { Modulus }\end{array}$ & $(\mathrm{GPa})$ \\
\hline \hline $\mathrm{c}_{11}$ & 10.59 \\
\hline $\mathrm{c}_{12}$ & 3.09 \\
\hline $\mathrm{c}_{13}$ & 4.11 \\
\hline $\mathrm{c}_{33}$ & 147.1 \\
\hline $\mathrm{c}_{44}$ & 5.97 \\
\hline
\end{tabular}




\section{FIGURE CAPTIONS}

FIG. 1 Early signal arrivals in the filtered, simulated acoustic waveform in aluminum plate.

FIG. 2 Pseudo Wigner-Ville distribution image and corresponding time domain signal for simulated acoustic waveform in aluminum plate.

FIG. 3 Group velocity of Lamb modes measured from PWVD analysis of a simulated waveform in aluminum plate compared to theoretical Lamb dispersion curves.

FIG. 4 Typical signal generated by a lead break source in a gr/ep plate identifying $\mathrm{S}_{0}$ and $\mathrm{A}_{0}$ Lamb mode components.

FIG. 5 Pseudo Wigner-Ville distribution images and corresponding time domain signals for propagation perpendicular to the fiber direction at distances of a) $8.89 \mathrm{~cm}$ and b) $15.24 \mathrm{~cm}$.

FIG. 6 Measured $\mathrm{S}_{0}$ and $\mathrm{A}_{0}$ mode group velocities and comparison with theoretical predictions (solid lines) for propagation perpendicular to the fiber direction.

FIG. 7 Measured $\mathrm{S}_{0}$ and $\mathrm{A}_{0}$ mode group velocities and comparison with theoretical predictions (solid lines) for propagation along the fiber direction. 
FIGURE 1

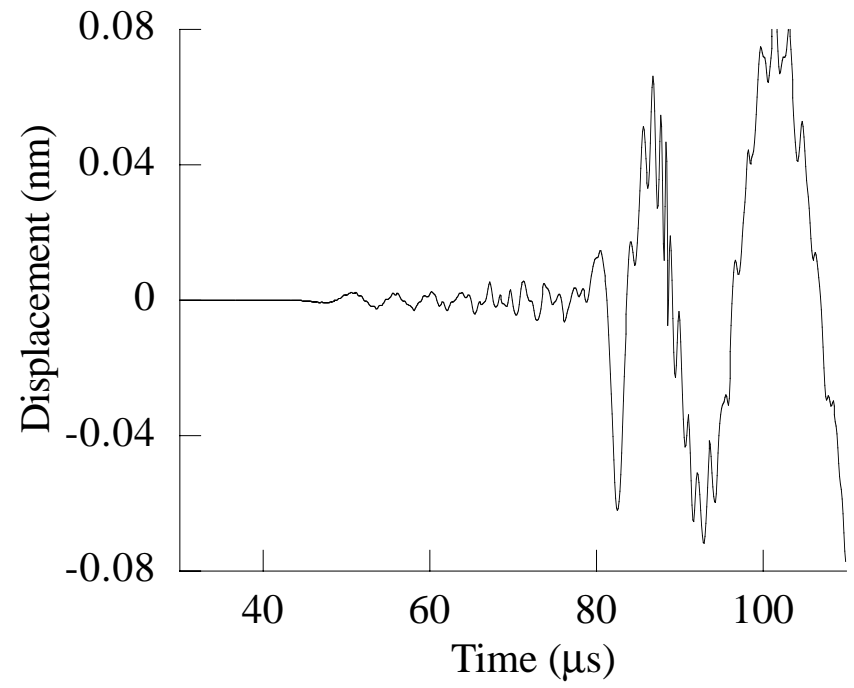

Journal of the Acoustical Society of America W. H. Prosser, M. D. Seale, and B. T. Smith 
FIGURE 2

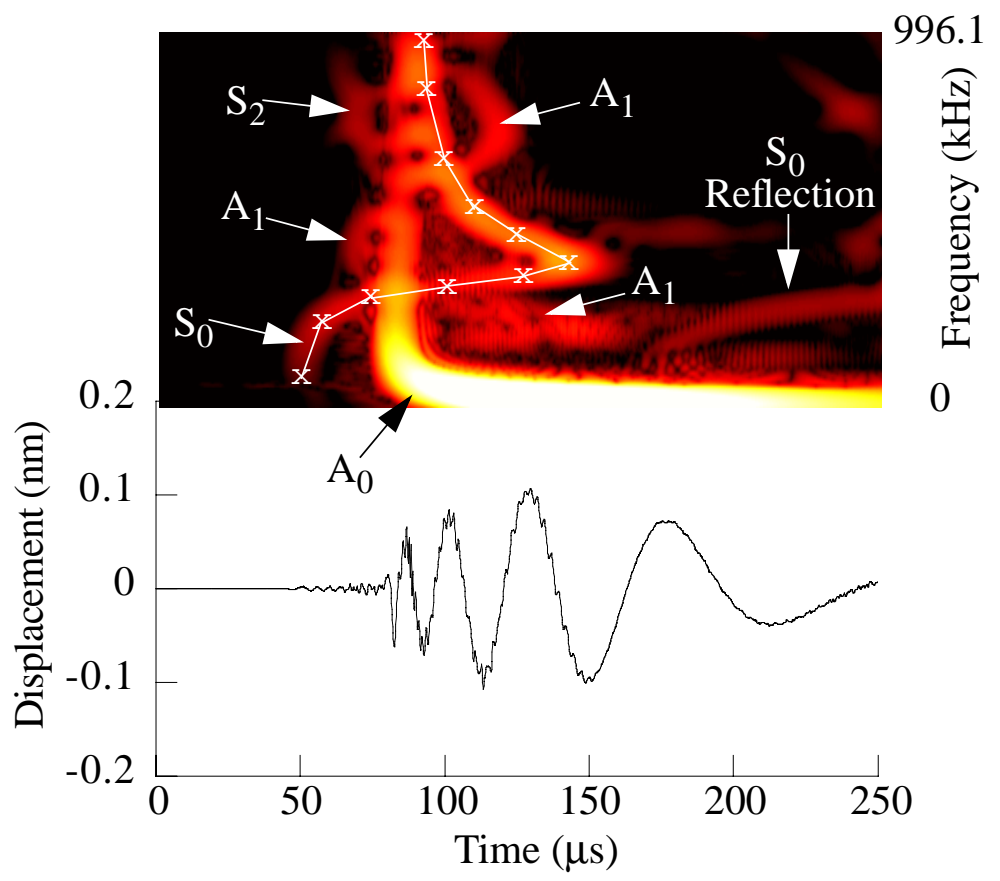


FIGURE 3

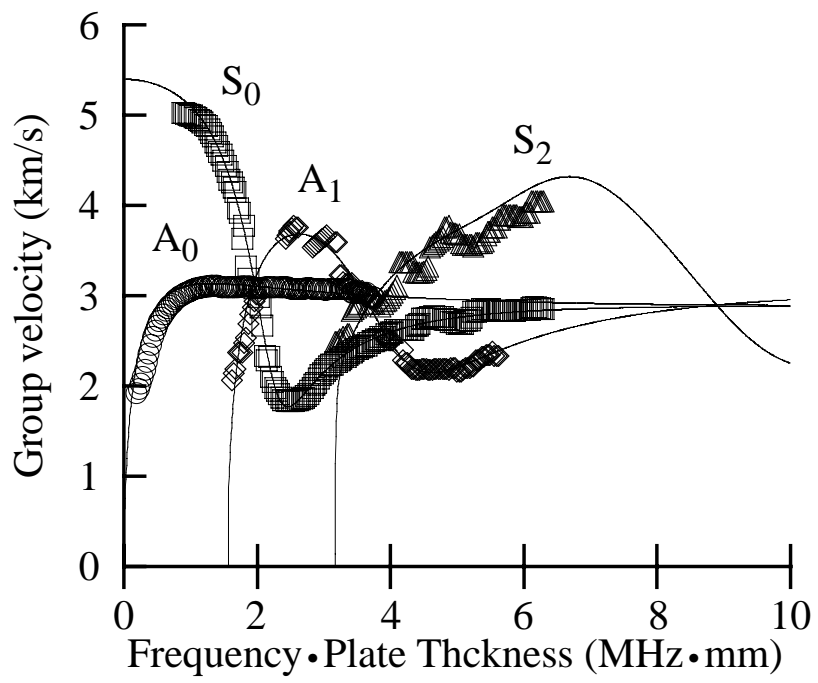

Journal of the Acoustical Society of America W. H. Prosser, M. D. Seale, and B. T. Smith 
FIGURE 4

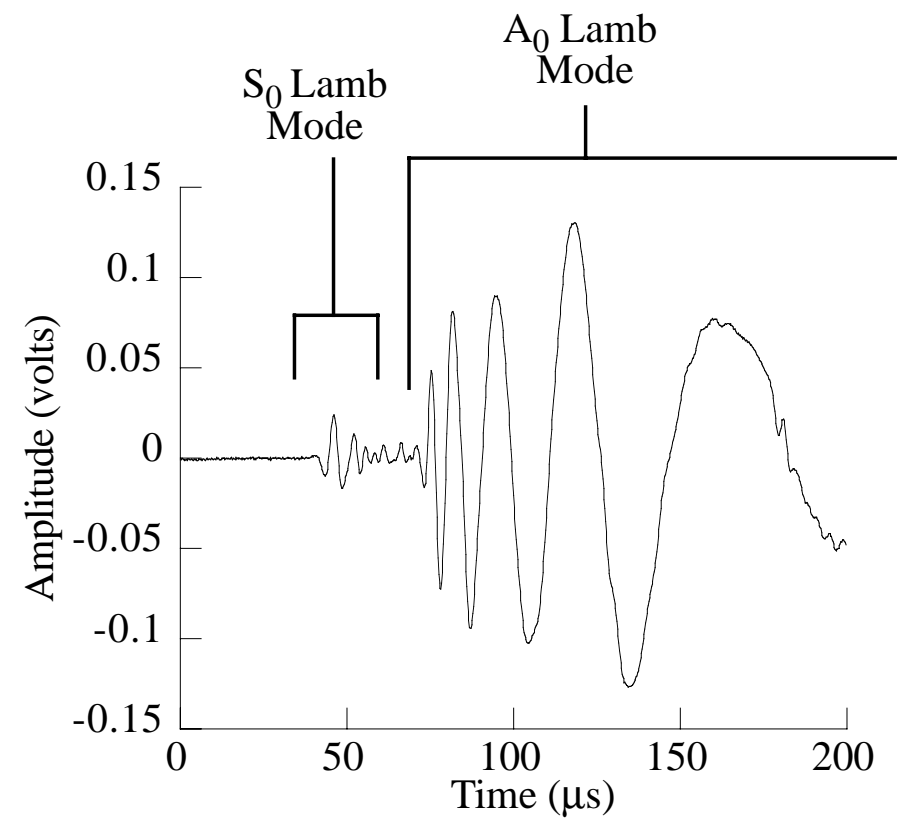

Journal of the Acoustical Society of America W. H. Prosser, M. D. Seale, and B. T. Smith 
FIGURE 5

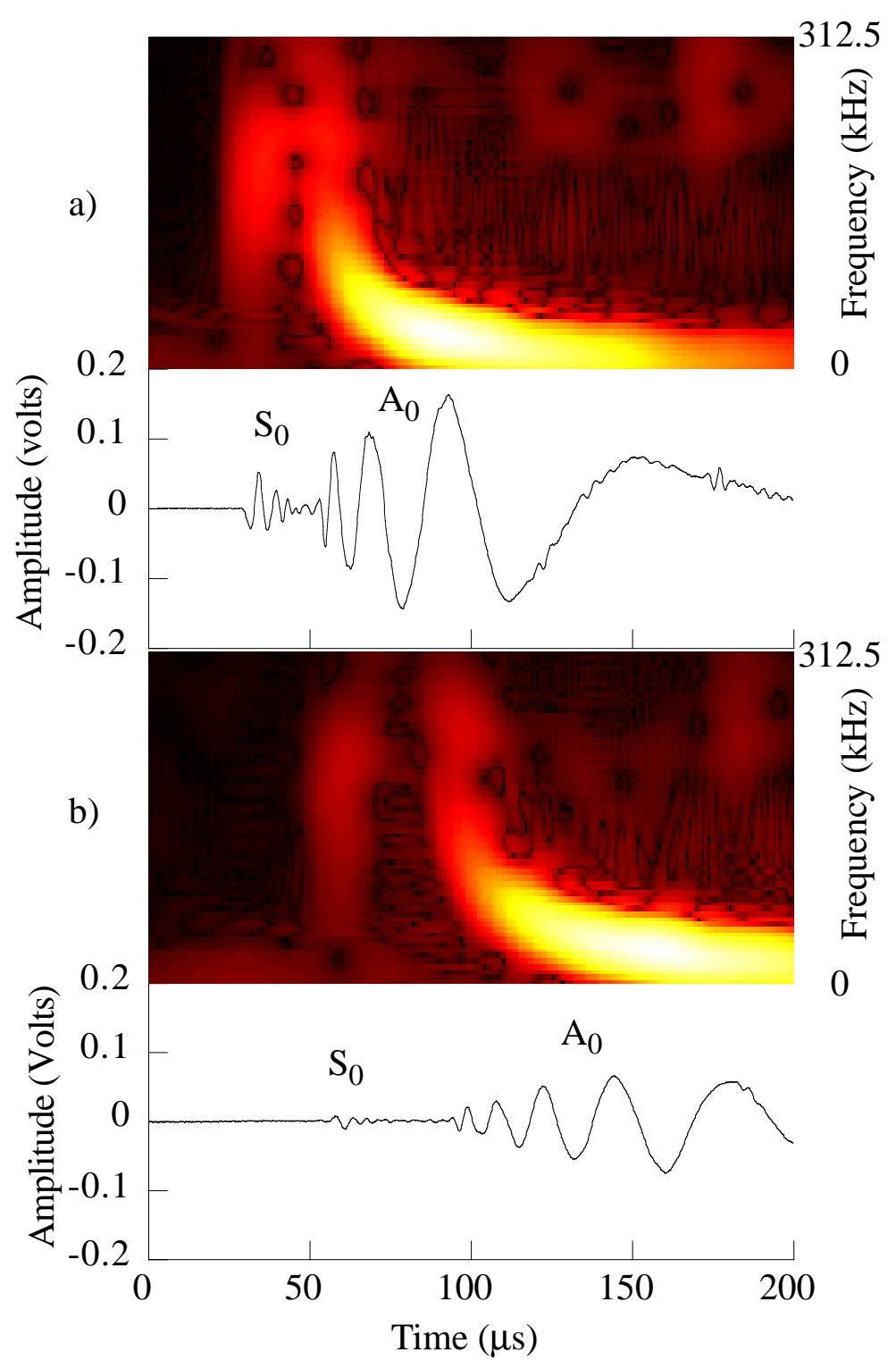

Journal of the Acoustical Society of America

W. H. Prosser, M. D. Seale, and B. T. Smith 
FIGURE 6

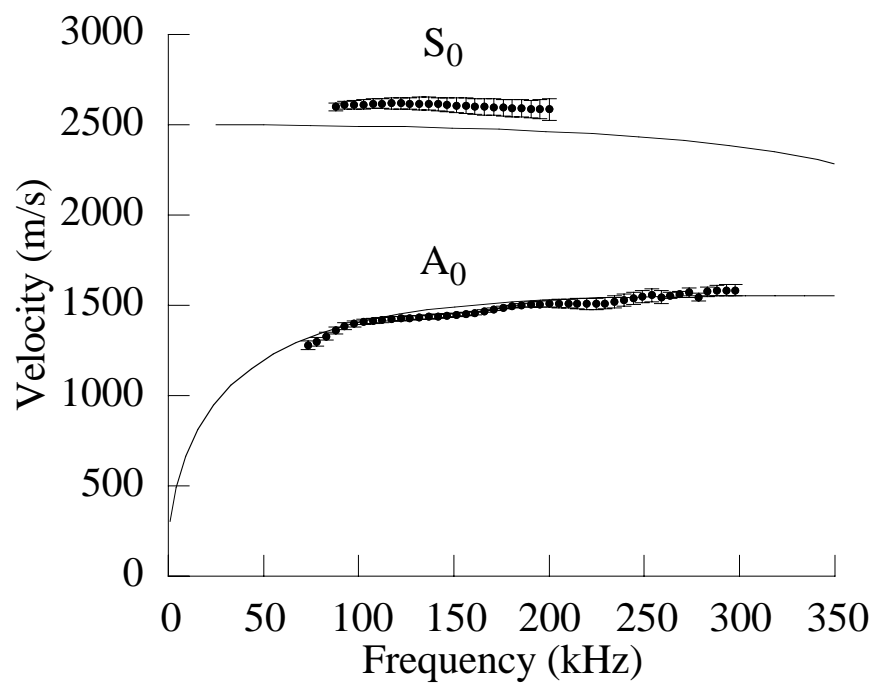

Journal of the Acoustical Society of America W. H. Prosser, M. D. Seale, and B. T. Smith 
FIGURE 7

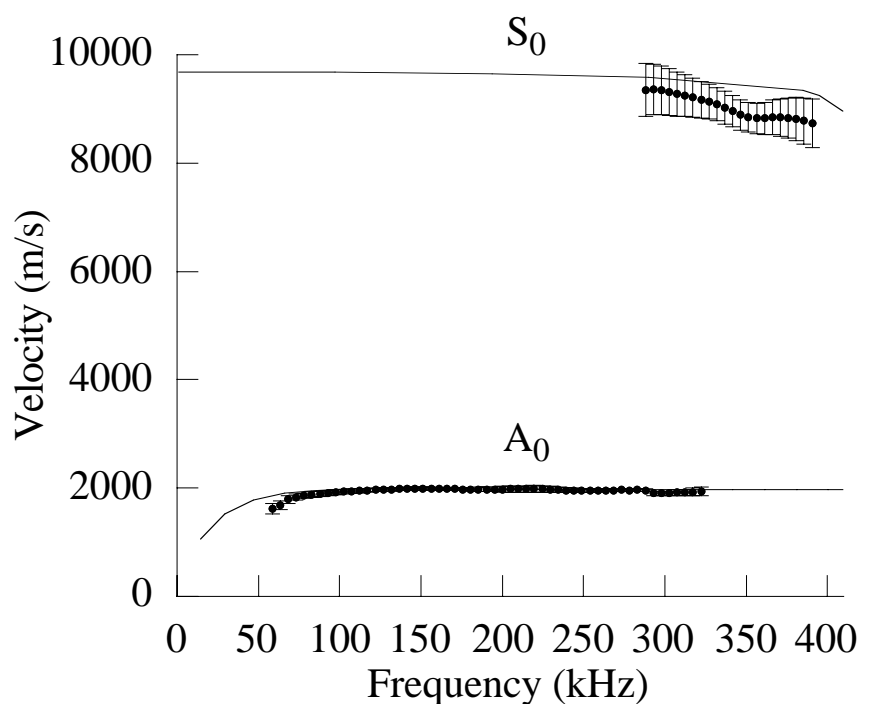

Journal of the Acoustical Society of America W. H. Prosser, M. D. Seale, and B. T. Smith 